\title{
Antenatal diagnosis of congenital heart disease and Down's syndrome: the potential effect on the practice of paediatric cardiology
}

\author{
Majd Abu-Harb, Jonathan Wyllie, Edmund Hey, Sam Richmond, Christopher Wren
}

\begin{abstract}
Objective-To predict the effect of antenatal ultrasound screening for congenital heart disease and maternal serum screening for Down's syndrome on the practice of paediatric cardiology and paediatric cardiac surgery.

Design-A retrospective and prospective ascertainment of all congenital heart disease diagnosed in infancy in 1985-1991.

Setting-One English health region.

Patients-All congenital heart disease diagnosed in infancy by echocardiography, cardiac catheterisation, surgery, or necropsy was classified as "complex", "significant", or "minor" and as "detectable" or "not detectable" on a routine antenatal ultrasound scan.
\end{abstract}

Results-1347 infants had congenital heart disease which was "complex" in $13 \%$, "significant" in $55 \%$, and "minor" in $32 \% .15 \%$ of cases were "detectable" on routine antenatal ultrasound. Assuming $20 \%$ detection and termination of $67 \%$ of affected pregnancies, liveborn congenital heart disease would be reduced by $2 \%$, infant mortality from congenital heart disease by $5 \%$, and paediatric cardiac surgical activity by $3 \%$. Maternal screening for Down's syndrome, assuming $75 \%$ uptake, $60 \%$ detection, and termination of all affected pregnancies, would reduce liveborn cases of Down's syndrome by $45 \%$, liveborn cases of congenital heart disease by $3.5 \%$, and cardiac surgery by $2 \cdot 6 \%$.

Conclusions-Screening for congenital heart disease using the four chamber view in routine obstetric examinations and maternal serum screening for Down's syndrome is likely to have only a small effect on the requirements for paediatric cardiology services and paediatric

Keywords: antenatal diagnosis; congenital heart dis-

Paediatric cardiology emerged as a recognised separate specialty about one generation ago. Since that time there has been rapid progress in the diagnosis and treatment of children with congenital heart disease, producing a profound improvement in their life expectancy and quality of life. The pace of development continues but because the specialty depends more and more upon expensive technology for both diagnosis and treatment, it is important to be able to predict the future requirements for treatment of patients with congenital heart disease in order to guarantee continued provision of services in an era of limited resources.

One recent development, which is likely to affect the demand for such services, is antenatal diagnosis of congenital heart disease which presents the possibility of termination of pregnancy in severe cases. Many of the more severe though less common abnormalities are potentially detectable in utero. Such patients are both more difficult and expensive to treat and have a lower expectation of survival and well-being. If the sensitivity and specificity of antenatal diagnosis were high and termination of pregnancy was more widely adopted, this would affect the practice of paediatric cardiology.

Recent speculation on the effect of antenatal diagnosis of congenital heart disease on paediatric cardiology and paediatric cardiac surgery has been made in the absence of data on the true spectrum of congenital heart disease. ${ }^{1-3}$ The aim of this study, therefore, was to examine the prevalence and spectrum of neonatal and infant congenital heart disease in a defined population, and to predict the effects of changes in practice (particularly termination of pregnancy because of congenital heart disease or Down's syndrome) on total infant mortality, on the prevalence at live birth and mortality of congenital heart disease, and on the requirement for cardiac surgery in infancy and beyond.

Epidemiological data for such a study will only be reliable if there is a well defined population with reliable registration of all births and deaths; a clearly identified referral route of all patients with congenital heart disease, preferably to a single centre; and complete ascertainment of all congenital heart disease, including babies who die and are diagnosed only at necropsy. ${ }^{4}$ These criteria are met in the Northern Region of England and allow this study to be performed with confidence in the findings. cardiac surgery.

(Br Heart f 1995;74:192-198) ease; Down's syndrome; paediatric cardiology

Department of
Paediatric Cardiology,
Freeman Hospital,
Newcastle upon Tyne
M Abu-Harb
J Wyllie
C Wren
Northern Regional
Fetal Abnormality
Survey, Newcastle
upon Tyne
E Hey
S Richmond
Correspondence to:
Dr Christopher Wren,
Department of Paediatric
Cardiology, Freeman
Hospital, Newcastle upon
Tyne NE7 7DN.
Accepted for publication
19 December 1994 


\section{Patients and methods}

\section{POPULATION}

The Northern Region of England is geographically well defined and has a population of 3076600 . There are about 40000 births each year and all cases of suspected congenital heart disease from 15 of the 16 health districts are referred to a single paediatric cardiology centre. Because patients from South Cumbria (population 172 500) are referred elsewhere for geographical reasons this health district was excluded from the study. Otherwise there is no outward cross border referral of infants with congenital heart disease.

\section{CASE ASCERTAINMENT}

Two well-known epidemiological surveys are based in the region. The Northern Regional Survey of Perinatal, Late Neonatal, and Infant Mortality was set up in 1981 to collect data on all perinatal and late neonatal deaths and was expanded in 1988 to include all infant deaths. ${ }^{5}$ The Northern Regional Fetal Abnormality Survey began as a pilot project in 1984 and was established in 1985 to collect data on all serious congenital malformations in fetuses, stillbirths, and liveborn infants and includes all cases of congenital heart disease diagnosed in infancy. ${ }^{6}$ The methods used in both surveys have been reported previously and are well validated.

All cases of congenital heart disease presenting in infancy in babies born in 1985-1991 were identified from the fetal abnormality survey. ${ }^{6}$ Cases identified only after death were identified from the perinatal mortality survey as described previously. ${ }^{4}$ Patients were included only if congenital heart disease was diagnosed before the first birthday and the diagnosis was confirmed by echocardiography, cardiac catheterisation, surgery, or necropsy.

Even though they do have congenital heart disease patients with an isolated cardiac arrhythmia, usually supraventricular tachycardia or complete atrioventricular block, were excluded from analysis to allow comparison with previous studies. Patients with patent ductus arteriosus complicating prematurity and those with dilated cardiomyopathy were excluded for the same reason. Patients with suspected but unconfirmed congenital heart disease were not included. Patients not referred until after 12 months of age were also excluded, even if congenital heart disease had been suspected in infancy by a referring paediatrician. The 13 cases in which antenatal diagnosis led to termination of pregnancy were also excluded from this analysis.

The two collaborative surveys also provided data on the regional birth rate, total infant mortality, intrauterine deaths after 20 weeks' gestation, all therapeutic terminations, all stillbirths, and all cases of Down's syndrome.

Data were collected on all antenatal deaths after 20 weeks' gestation, whether resulting from intrauterine death, therapeutic termination, or stillbirth. However, despite a rela- tively high overall necropsy rate in such cases in the perinatal mortality survey it became apparent that such necropsies as there were could not be relied upon to detect all congenital cardiac abnormalities. Unfortunately, examination of the data published in the few reports that were population based proved to be insufficient to estimate the prevalence of congenital heart disease in babies in our population who were not live born..$^{7-9}$ Antenatal deaths probably include a higher prevalence of cardiac malformations than live births. ${ }^{7-9}$ Thus diagnosis of heart defects in babies who will die in utero will reduce the effect of intervention based on recognition of congenital heart disease by 20 weeks' gestation. If the published data ${ }^{7-9}$ were applied to our population, they would suggest that antenatal mortality is higher for "significant" than for "complex" abnormalities and for "not detectable" rather than "detectable" malformations, further reducing the impact of antenatal ultrasound screening.

\section{SURVEY OF SURGICAL ACTIVITY}

A survey of all cardiothoracic surgery performed on patients under the age of 16 years in 1991-1992 yielded data on type of surgery (open or closed) in infancy and beyond and was correlated with the cardiac diagnosis. These years were chosen because changes in personnel and surgical practice in previous years meant the data from before 1991 were not representative.

\section{CLASSIFICATION OF SEVERITY}

Each cardiac abnormality was classified as "complex", "significant", or "minor" using a classification described previously." Congenital heart disease was taken to be "complex" when a valve or chamber was atretic or hypoplastic. Abnormalities were described as "significant" when four chambers of normal or increased size and four valves were present but intervention was required. Abnormalities were designated "minor" when no treatment was required. The latter group comprised mainly small ventricular septal defects and relatively mild aortic or pulmonary valve stenosis. Exceptions to this classification included endocardial fibroelastosis (which was classified as "complex") and complete atrioventricular septal defect (designated "significant"). Patients with multiple cardiac abnormalities were classified according to the most severe diagnosis. For example, a baby with coarctation of the aorta and double inlet left ventricle would be classified under "complex" because of the latter diagnosis, even though the former had precipitated presentation.

Any method of classification of congenital heart disease that attempts to be simple will be imperfect. Our classification of severity, based on diagnosis, has been described previously $^{4}$ and is well validated by the relation between classification and outcome (see below and fig 1). This approach seems more logical than classifications based on surgical risk, ${ }^{1}$ which varies greatly between institutions 


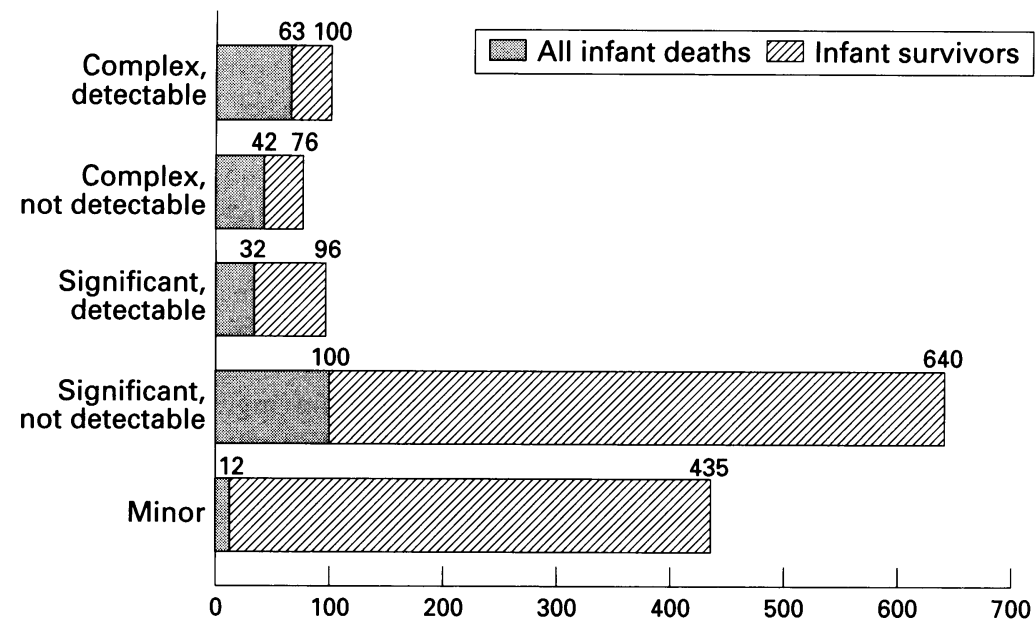

Figure 1 Number of confirmed cases of congenital heart disease in infancy, classified as "complex", "significant", or "minor" and as "detectable" or "not detectable" (see text for explanation). such conditions can be detected. However, most ventricular septal defects are not large, most aortic or pulmonary stenosis is not severe, and most easily detectable coarctations are associated with a more major abnormality of the heart itself which will define the detectability. The natural history of ventricular septal defects in fetal life is not clear but because they would not be regarded in most centres as an indication for termination of pregnancy they would not have a great effect on our calculations.

Because of the rapid change in obstetric practice between 1985 and 1991 it was not possible to ascertain the number of cases of congenital heart disease in our population which resulted from pregnancies in which four chamber screening had been used. In the early years of the study there was no prospective four chamber screening but it had been introduced in several of the larger maternity units by the late 1980 s. $^{14}$

and will diminish with advances in surgical treatment.

\section{DEFINING ANTENATAL DETECTABILITY}

Each cardiac abnormality was also classified as "detectable" or "not detectable" (table 1). Detectability assumes that screening is being performed by an obstetric sonographer as part of a routine antenatal assessment incorporating a four chamber view of the heart at around 18 weeks' gestation. Such screening should identify abnormalities which produce marked cardiomegaly, an obvious abnormality of the atrioventricular connection, or a disparity between the sizes of the atria or ventricles or both. ${ }^{310}$ There are reports of most cardiac malformations being detected. However, for many of the more common abnormalities, those cases that are detected are at the severe end of the spectrum. ${ }^{11}$ It is widely recognised that screening based on the four chamber view may fail to identify some types of major malformations ("complex" in our classification) affecting the left and right heart outflows. ${ }^{31213}$ Our classification of detectability is similar to that described by others, ${ }^{310}$ but for the sake of simplicity we have considered as "undetectable" all cases of ventricular septal defect, pulmonary stenosis, aortic stenosis, and coarctation of the aorta. This is not to deny that some severe cases of

\section{Results}

In the seven years 1985-1991 there were 269335 live births in the 15 health districts (population 2904100 ), a birth rate of 13.2 per 1000 population. There were 2206 infant deaths, producing a total infant mortality of 8.17 per 1000 live births. In the same period, 1347 cases of congenital heart disease were confirmed in infancy, a prevalence at live birth of 5.0 per 1000 live births. Of these 1347 infants, 230 died before their first birthday from their cardiac abnormalities, an infant cardiac mortality rate of $17 \%$. In 164 $(71 \%)$ cases, congenital heart disease had been diagnosed in life, whereas in 66 (29\%) the diagnosis was only made at necropsy. Nineteen babies with congenital heart disease died in the first year of life for reasons unconnected with their cardiac abnormalities.

\section{CLASSIFICATION}

Classified by severity, $13 \%$ of the infants had "complex" heart disease, 55\% had "significant" heart disease, and $32 \%$ had "minor" heart disease (fig 1). This classification of severity is validated by the cardiac mortality in infancy in each group, which ranged from $59 \%$ in "complex" congenital heart disease

Table 1 Classification of common congenital cardiovascular malformations

\begin{tabular}{|c|c|c|}
\hline & "Detectable" & "Undetectable" \\
\hline "Complex" & $\begin{array}{l}\text { Hypoplastic left heart } \\
\text { Mitral atresia } \\
\text { Tricuspid atresia } \\
\text { Pulmonary atresia/intact septum } \\
\text { Double inlet ventricle } \\
\text { Ebstein/tricuspid valve dysplasia }\end{array}$ & $\begin{array}{l}\text { Truncus arteriosus } \\
\text { Pulmonary atresia with ventricular septal defect } \\
\text { Double outlet ventricle }\end{array}$ \\
\hline "Significant" & $\begin{array}{l}\text { Complete atrioventricular septal defect } \\
\text { Partial atrioventricular septal defect }\end{array}$ & $\begin{array}{l}\text { Large ventricular septal defect } \\
\text { Atrial septal defect } \\
\text { Patent ductus } \\
\text { Severe aortic stenosis } \\
\text { Severe pulmonary stenosis } \\
\text { Transposition of the great arteries } \\
\text { Coarctation of the aorta } \\
\text { Total anomalous pulmonary venous connection } \\
\text { Tetralogy of Fallot }\end{array}$ \\
\hline "Minor" & & $\begin{array}{l}\text { Small ventricular septal defect } \\
\text { Mild pulmonary stenosis } \\
\text { Mild aortic stenosis }\end{array}$ \\
\hline
\end{tabular}




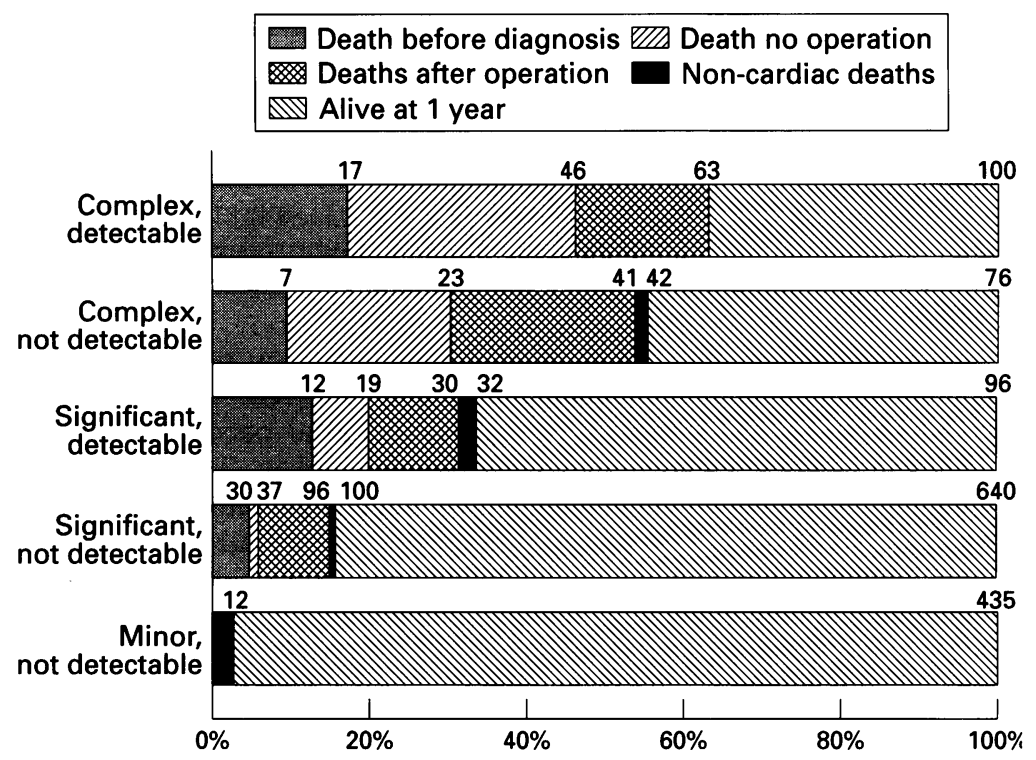

Figure 2 Outcome of all cases of congenital heart disease confirmed in infancy.

to $0 \%$ in "minor" heart disease (fig 1). "Complex" heart disease contributed $45 \%$ of all deaths, even though it accounted for only $13 \%$ of all cases.

Fifteen percent (196) of cases were detectable by routine antenatal screening. The correlation between detectability and severity was not good as only 100 of 176 (57\%) “complex" abnormalities were detectable and only 100 of $196(51 \%)$ of detectable abnormalities were "complex" (fig 2 ). The outcome was affected mainly by the severity rather than detectability. Cardiac mortality in the "complex" group was $63 \%$ for detectable malformations and $54 \%$ for non-detectable malformations. However, infant cardiac mortality in "significant" heart disease was $31 \%$ for detectable abnormalities and only $15 \%$ for non-detectable abnormalities, the difference mainly accounted for by the high proportion of complete atrioventricular septal defects, often associated with chromosomal abnormalities, in the "significant" and detectable group (fig 2).

PREDICTION OF THE EFFECT OF ANTENATAL DIAGNOSIS OF CONGENITAL HEART DISEASE The effect of antenatal diagnosis of congenital heart disease depends upon the proportion of "detectable" cases, the number of such cases actually detected, and the number of terminations after diagnosis. The above data on the prevalence and spectrum of congenital heart disease in an infant population enable predic-

Table 2 Predicted effect of antenatal ultrasound screening assuming a $20 \%$ detection rate and termination of $67 \%$ of affected pregnancies

\begin{tabular}{lllc}
\hline Variable & Reduction (\%) & $\begin{array}{l}\text { Reduction per } \\
100 \text { 000 live } \\
\text { births }\end{array}$ & $\begin{array}{l}\text { Annual reduction } \\
\text { in UK }\end{array}$ \\
\hline Prevalence at livebirth of CHD & $2 \cdot 0$ & 10 & 70 \\
Infant mortality from CHD & $5 \cdot 4$ & $4 \cdot 5$ & 33 \\
Total infant mortality & $0 \cdot 6$ & $4 \cdot 5$ & 33 \\
Survival beyond 1 yr with CHD & $1 \cdot 2$ & 5 & 36 \\
Paediatric cardiac surgery & $2 \cdot 9$ & 17 & 120 \\
Prevalence of Down's at livebirth & $2 \cdot 3$ & 3 & 20 \\
\hline
\end{tabular}

$\mathrm{CHD}$, congenital heart disease. tions to be made about the effect of changes in the detection rate and the termination rate on several aspects of congenital heart disease. We have used this model to investigate the effect of antenatal diagnosis on: ( $a$ ) the prevalence at live birth of congenital heart disease, (b) infant mortality from congenital heart disease, (c) total infant mortality, (d) paediatric cardiac surgical activity, $(e)$ the prevalence at live birth of Down's syndrome.

Effect on prevalence at live birth

As shown above, $15 \%$ of all congenital heart disease is detectable on an antenatal four chamber ultrasound scan. Therefore, if all these abnormalities were detected and all affected pregnancies were terminated, the prevalence at live birth of congenital heart disease would be reduced by $15 \%$ (fig 3 ). However, the detection rate in most centres in the United Kingdom is around $20 \%$ of detectable cases $^{14}$ and in only about two thirds of detected cases is the pregnancy terminated. ${ }^{315} 16$ This means that the prevalence at live birth is reduced by only $2 \%(15 \% \times$ $20 \% \times 67 \%$ ) (fig 3 and table 2 ). Even if the detection rate could be doubled to $40 \%$, the prevalence at live birth would still be reduced by only $4 \%$.

\section{Effect on infant mortality from congenital heart disease}

The mortality from congenital heart disease in infancy in this population was $17 \%$. Sixty six deaths (29\%) occurred before recognition of congenital heart disease, another $59(26 \%)$ in the absence of surgery, and $105(46 \%)$ despite surgery. Ninety three (40\%) of 230 deaths were in babies with detectable cardiac malformations (fig 2). Antenatal detection of $20 \%$ of such cases and termination of $67 \%$ of those detected would reduce infant cardiac mortality by $5 \cdot 4 \%$ (table 2 ) and would reduce deaths from "complex" heart disease by only $8 \cdot 1 \%$. However, such a policy would also reduce survivors at one year by $1 \cdot 2 \%$.

\section{Effect on total infant mortality}

The infant mortality in the Northern Region is 8.17 per 1000 live births. Congenital heart disease accounts for $10.4 \%$ of all infant deaths and the cardiac abnormalities in babies who died were detectable in $40 \%$. Detection of $20 \%$ of detectable malformations and termination of $67 \%$ of these pregnancies would reduce the total infant mortality by $0.6 \%$-that is, from $8 \cdot 17$ to $8 \cdot 12$ per 1000 (table 2).

Effect on paediatric cardiac surgical activity During the two years 1991-1992 there were 442 operations, of which 215 (49\%) were performed in infancy (fig 4). Infant surgery comprised $117(26 \%)$ open and $96(22 \%)$ closed operations while beyond infancy there were $181(41 \%)$ open and $48(11 \%)$ closed operations. Obviously some surgery was carried out on children whose congenital heart disease is recognised only after infancy so it is not possible, at present, to relate the surgical 


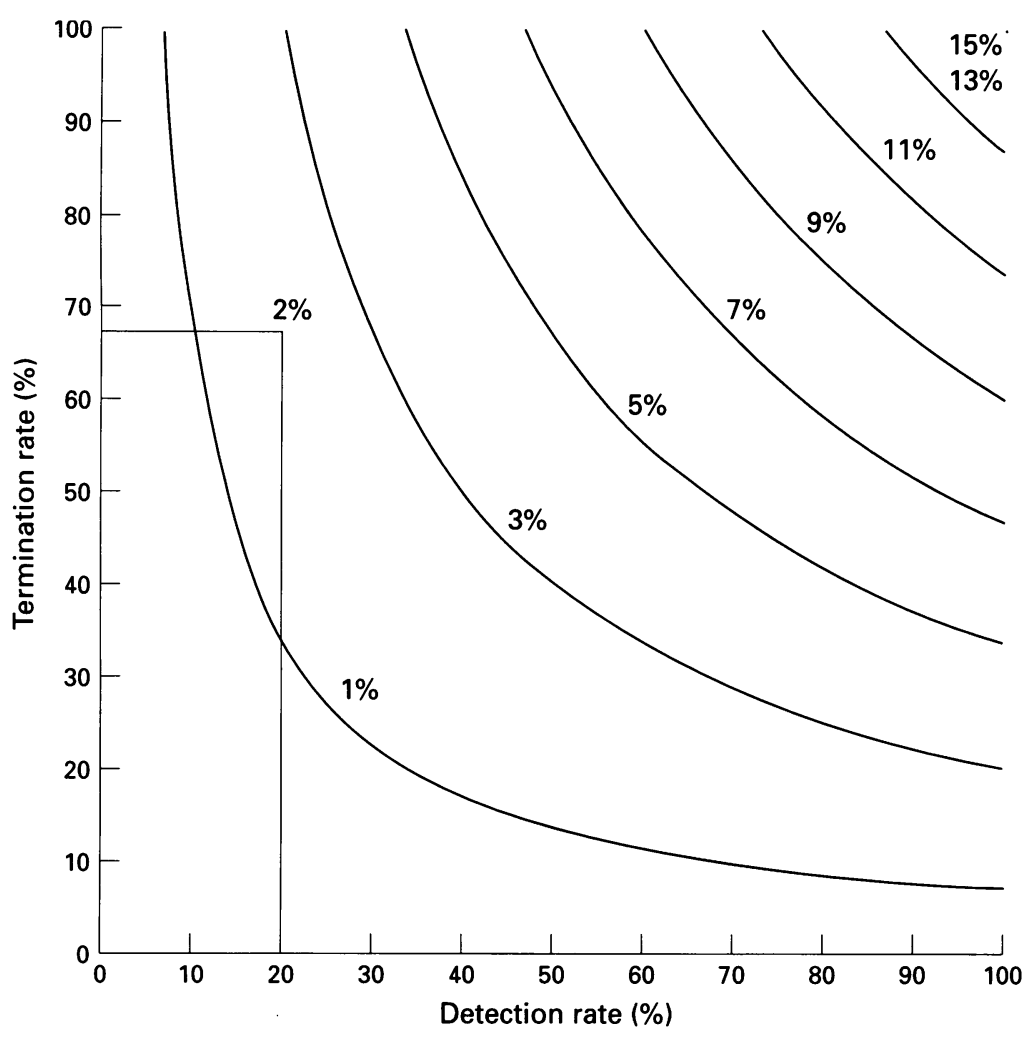

Figure 3 Cumulative effect of detection rate and termination rate on the prevalence at live birth of congenital heart disease. $15 \%$ of all cases were "detectable" so a detection rate of $100 \%$ plus a termination rate of $100 \%$ would reduce live born congenital heart disease by $15 \%$. Assuming 20\% of "detectable" cases are detected and two thirds of affected pregnancies are terminated, the reduction in live born congenital heart disease would be $2 \%$. The effect of any detection rate with any termination rate can be calculated from this graph.

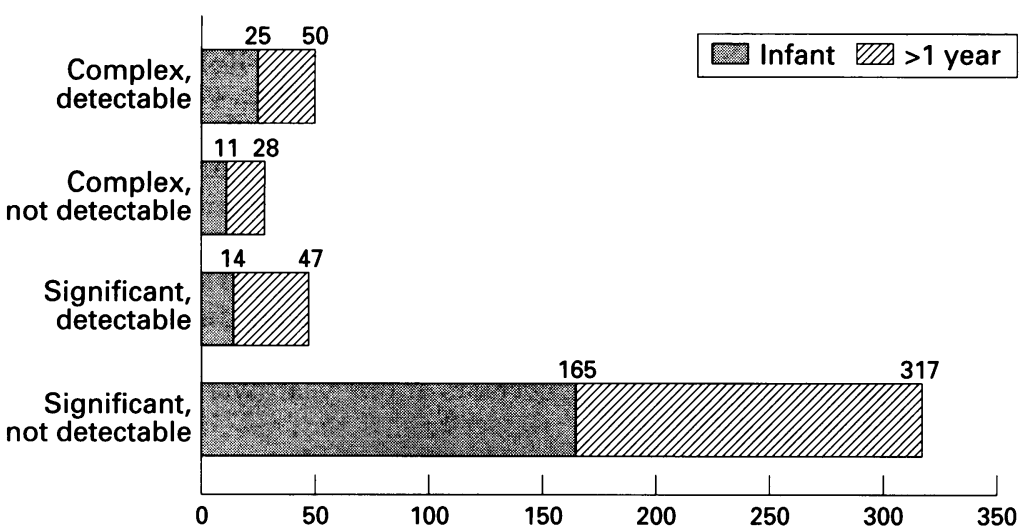

Figure 4 Numbers of paediatric cardiac surgical operations according to the classification groups. Stippled, surgery in infancy; hatched, surgery between one and 16 years. As can be seen, most surgery is performed on infants and children with "significant" and "nondetectable" malformations. for $22 \%$ of all surgery and $26 \%$ of open heart surgery. The numbers of operations per patient were very similar for "complex" and "significant" heart disease and for "detectable" and "not detectable" abnormalities. Twenty six (6\%) operations were in patients with Down's syndrome, $15(58 \%)$ of whom had "detectable" cardiac abnormalities.

Assuming a $20 \%$ detection rate on antenatal screening and a $67 \%$ termination rate of affected pregnancies, total paediatric cardiac surgery would be reduced by $2.9 \%$ (table 2 ), infant surgery by $2.4 \%$, open heart surgery by $3.5 \%$, and infant open heart surgery by $2.8 \%$.

\section{Effect on Down's syndrome}

In the seven years 1985-1991 there were 415 cases of Down's syndrome in the 15 health districts of the Northern Region. Of these, 76 $(18 \%)$ were detected antenatally and the pregnancies were terminated and $23(6 \%)$ were stillborn. Thus, 315 were live born $(1 \cdot 2$ per 1000 live births) and 106 (34\%) of these had congenital heart disease, accounting for $8 \%$ of all congenital heart disease. More than half $(52 \%)$ of their cardiac malformations were detectable, being either complete or partial atrioventricular septal defects. Other malformations encountered were ventricular septal defect (25\%), atrial septal defect (7\%), patent ductus arteriosus (9\%), tetralogy of Fallot (4\%), and coarctation of the aorta $(2 \%)$. Thus none of the cardiac abnormalities in Down's syndrome was classified as "complex". Fifteen (14\%) babies with Down's syndrome and congenital heart disease died (all with complete atrioventricular septal defect).

Assuming a $20 \%$ detection rate and a $67 \%$ termination rate, the prevalence at live birth of Down's syndrome would be reduced by only $2.3 \%$ (table 2 ). However, in practice both the detection rate and the termination rate might be expected to be slightly higher as more careful examination of the heart might be indicated in some cases by a finding such as duodenal atresia and the antenatal diagnosis of a complete atrioventricular septal defect strongly suggests the possibility of Down's syndrome and, therefore, may be a stronger indication for termination of pregnancy, even in the absence of chromosomal confirmation of the diagnosis.

\section{EFFECT OF MATERNAL BLOOD TESTING} SCREENING FOR DOWN'S SYNDROME

Maternal blood testing has been adopted in several regions for antenatal detection of Down's syndrome ${ }^{1718}$ and can probably detect up to $60 \%$ of cases of Down's syndrome. ${ }^{19-21}$ Assuming that $75 \%$ of mothers undergo testing and $100 \%$ of those with a positive test elect for termination, this would reduce the number of children born with Down's syndrome by $45 \%$ and on its own would reduce the prevalence at live birth of congenital heart disease by $3.6 \%$ (table 3 ). (It would probably be more realistic to assume $75 \%$ uptake, $50 \%$ detection, and $75 \%$ termination, ${ }^{19}$ which would reduce the overall diac surgical activity.

Detectable abnormalities (which comprise $21 \%$ of congenital heart disease in the "complex" and "significant" categories) accounted 
Table 3 Predicted effect of maternal serum screening for Down's syndrome

\begin{tabular}{lcll}
\hline Variable & (\%) Reduction & $\begin{array}{l}\text { Reduction per } \\
100 \text { 000 live births }\end{array}$ & $\begin{array}{l}\text { Annual reduction } \\
\text { in UK }\end{array}$ \\
\hline Prevalence at livebirth of CHD & $3 \cdot 5$ & 18 & 130 \\
Infant mortality from CHD & $2 \cdot 9$ & $2 \cdot 5$ & 18 \\
Total infant mortality & $0 \cdot 3$ & $2 \cdot 5$ & 18 \\
Survival beyond 1 yr with CHD & $3 \cdot 9$ & $15 \cdot 5$ & 112 \\
Paediatric cardiac surgery & $2 \cdot 6$ & 15 & 109 \\
Prevalence of Down's at lifebirth & $45 \cdot 0$ & 50 & 357 \\
\hline
\end{tabular}

CHD, congenital heart disease. port, and on the quality of the ultrasound equipment. The success in finding "detectable" abnormalities can be quite high ${ }^{31013}$ although such reports usually involve selfselecting participating centres and have no identified population base and incomplete ascertainment of false negative diagnoses. A more realistic estimate of the present detection rate of "detectable" malformations in most paediatric cardiology units in the United Kingdom is around $20 \%{ }^{14}$ and for this reason our calculations have been based on this figure. However, if detection rates are thought to be higher or lower, the calculations can easily be adjusted accordingly. A recent review concluded that there is a limit to how far screening for congenital heart disease can go using the four chamber view of the fetal heart in a routine obstetric ultrasound scan, partly because of the reliance on non specialist sonographers and partly because it is limited to the four chamber view. ${ }^{12}$ It would be possible to increase the detection rate substantially by using specialist sonographers and complete ultrasound examination of the heart but such a policy would probably be very expensive. Fermont $e t$ al estimate the need for one specialist sonographer and one additional ultrasound machine for each 900 pregnancies in the population. ${ }^{12}$ This would imply an extra 800 sonographers and machines in the United Kingdom - an enormous allocation of resources which is difficult to justify given the good prognosis for most congenital heart disease (fig 1). The combined prevalence of "complex" heart disease and atrioventricular septal defect in Down's syndrome in our study is 1 in 1140 live births. This suggests that each full-time expert sonographer would expect to encounter one case every 15 months.

The detection of congenital heart disease is obviously not the main reason for screening for Down's syndrome. However, given the high prevalence of cardiac malformations in Down's syndrome ( $36 \%$ in this series) any change in practice that reduces the number of live born babies with Down's syndrome will also reduce the birth prevalence of congenital heart disease. Screening for Down's syndrome is patchy at present ${ }^{17}$ but is likely to be more widely introduced. ${ }^{18}$ "Demonstration projects" have shown that up to $60 \%$ of cases can be detected ${ }^{1920}$ but the overall detection rate in a population is also affected by the uptake of the test, the proportion of positive tests proceeding to amniocentesis, and the proportion of affected pregnancies terminated. As each of these is likely to be less than $100 \%$ they will further reduce the performance of the screening test. Our calculations have been based on the most optimistic forecasts $^{1920}$ and show that a $60 \%$ detection rate for the screening test is likely, at best, to detect $45 \%$ of cases of Down's syndrome, reducing liveborn congenital heart disease by $3.6 \%$ (table 3 ). If we used current rather than forecast figures, ${ }^{19}$ only $28 \%$ of Down's syndrome pregnancies would be terminated, reducing the birth prevalence of congenital and has relied on the four chamber view of the fetal heart. The detection rate obviously depends on the expertise and enthusiasm of the sonographer, on expert input and sup- 
heart disease by $2 \cdot 3 \%$. As can be seen by comparing tables 2 and 3 , screening for Down's syndrome will have a proportionately smaller effect on mortality from congenital heart disease and on requirements for cardiac surgery than ultrasound screening for congenital heart disease, as the types of cardiac malformation encountered in Down's syndrome are usually less severe and none of the cases in this series was classified as "complex".

If our data are extrapolated to the whole of the United Kingdom they suggest that if the detection rate of antenatal ultrasound screening for congenital heart disease could be doubled (from $20 \%$ to $40 \%$ ) this would translate into a further reduction of 70 live born cases of congenital heart disease, 33 fewer infant deaths from congenital heart disease, 120 fewer operations for congenital heart disease, 22 fewer live born babies with Down's syndrome, and 36 fewer survivors beyond infancy each year (table 3 ). This applies to a population of 55000000 with 715000 live births each year. Our model permits the likely effect of any change in detection rate or termination rate to be calculated. Although it is currently impossible to cost many aspects of the antenatal detection of congenital heart disease, it is important that allocation of resources to a screening programme should only be increased if it can be shown to be cost effective and of overall benefit. ${ }^{23}$

We thank the Steering Groups of the Northern Regional Survey of Perinatal, Neonatal, and Late Infant Mortality and the Northern Regional Fetal Abnormality Survey for acces to their data and to the Northern Regional Health Authority (M A-H) and the Sir Jules Thorne Charitable Trust (JPW) for financial support.

1 Cullen S, Sharland GK, Allan LD, Sullivan ID. Potential impact of population screening for prenatal diagnosis of congenital heart disease. Arch Dis Child 1992;67:775-8.

2 Achiron R, Glaser J, Gelernter I, Hegesh J, Yagel S. Extended fetal echocardiographic examination for detecting cardiac malformations in low risk pregnancies. $B M \mathcal{F}$ 1992;304:671-4.

3 Sharland GK, Allan LD. Screening for congenital heart disease prenatally. Results of a $2 \frac{1}{2}$-year study in the South East Thames Region. Br $\mathcal{F}$ Obstet Gynaecol 1992 99:220-5.

4 Abu Harb M, Hey E, Wren C. Death in infancy from unrecognised congenital heart disease. Arch Dis Child 1994;71:3-7.

5 Northern Regional Health Authority Co-ordinating Group. Perinatal mortality: a continuing collaborative regional survey. $B M \mathcal{A} 1984 ; 288$ :1717-20.

6 Northern Regional Survey Steering Group. Fetal abnormality: an audit of its recognition and management. Arch Dis Child 1992;67:770-4.

7 Mitchell SC, Korones SB, Berendes HW. Congenital heart disease in 56,109 births. Circulation 1971;43: heart disea.

8 Bound JP, Logan WFWE. Incidence of congenital heart disease in Blackpool 1957-1971. Br Heart $\mathcal{f} 1977 ; 39$ 445-50.

9 Hoffman JIE, Christianson R. Congenital heart disease in a cohort of 19,502 births with long term follow-up. $\mathrm{Am}$ f Cardiol 1978;42:641-7.

10 Copel JA, Pilu G, Green J, Hobbins JC, Kleinman CS. Fetal echocardiographic screening for congenital heart disease: The importance of the four-chamber view. $A m$ disease: The importance of the four

11 Sharland GK, Lockhart SM, Chita SK, Allan LD. Factors influencing the outcome of congenital heart disease detected prenatally. Arch Dis Child 1990;65:284-87.

12 Fermont L, Kachaner J, Sidi D. Detection of congenital heart disease: why and how to screen a fetal population. In: Chervenak FA, Isaacson GC, Campbell S, eds. Ultrasound in obstetrics and gynaecology. Boston: Little, Brown \& Co, 1993;115-21

13 Bromley B, Estroff JA, Sanders SP, Parad R, Roberts D, Frigoletto FD, et al. Fetal echocardiography: Accuracy and limitations in a population at high and low risk for and limitations in a population at high and low risk for

14 Wyllie JP, Wren C, Hunter S. Screening for fetal cardiac malformations. Br Heart $\mathcal{F}$ 1994;71 (April):20-7.

15 Smythe JF, Copel JA, Kleinman CS. Outcome of prenatally detected cardiac malformations. $A m \mathcal{F}$ Cardio 1992;69:1471-4.

16 Allan LD. Congenital heart disease. In: Brock DJH, Rodeck $\mathrm{CH}$, Ferguson-Smith MA, eds. Prenatal diagnosis and screening. Edinburgh: Churchill Livingstone, $1992 ; 271-85$.

17 Wald N, Wald K, Smith D. The extent of Down's syndrome screening in Britain in 1991. Lancet 1992;40: 492.

18 Connor M. Biochemical screening for Down's syndrome. BMF 1993;306:1705.

19 Wald NJ, Kennard A, Densem JW, Cuckle HS, Chard T, Butler L. Antenatal maternal serum screening for Down's syndrome: results of a demonstration project. $B M \mathcal{F}$ 1992;305:391-4

20 Haddow JE, Palomaki GE, Knight GJ, Williams J, Pulkkinen A, Canick JA, et al. Prenatal screening for Down's syndrome with use of maternal serum markers. N Engl F Med 1992;327:588-93.

21 Wald NJ, Kennard A. Prenatal biochemical screening for Down's syndrome and neural tube defects. Curr Opin Obstet Gynecol 1992;4:302-7.

22 Members of the Joint Study Group on Fetal Abnormalities. Recognition and management of fetal Abnormalities. Recognition and management

23 Ades AE. Evaluating screening tests and screening programmes. Arch Dis Child 1990;65:792-5. 